OPEN ACCESS

Edited by:

Marcos Ferreira Minicucci, Sao Paulo State University, Brazil

Reviewed by:

Suzana Erico Tanni, Sao Paulo State University, Brazi Huaiwu He,

Peking Union Medical College Hospital (CAMS), China

*Correspondence: Meng-Rui Lee leemr@ntu.edu.tw

Specialty section: This article was submitted to Intensive Care Medicine and Anesthesiology, a section of the journal Frontiers in Medicine

Received: 02 March 2021 Accepted: 12 May 2021

Published: 02 June 2021

Citation:

Keng L-T, Liang S-K, Tseng C-P, Wen Y-F, Tsou P-H, Chang C-H, Chang L-Y, Yu K-L, Lee M-R and Ko J-C (2021) Functional Status After

Pulmonary Rehabilitation as a Predictor of Weaning Success and

Survival in Patients Requiring Prolonged Mechanical Ventilation.

Front. Med. 8:675103.

doi: 10.3389/fmed.2021.675103

\section{Functional Status After Pulmonary Rehabilitation as a Predictor of Weaning Success and Survival in Patients Requiring Prolonged Mechanical Ventilation}

\author{
Li-Ta Keng ${ }^{1}$, Sheng-Kai Liang ${ }^{1}$, Chi-Ping Tseng ${ }^{2}$, Yueh-Feng Wen ${ }^{1}$, Ping-Hsien Tsou ${ }^{1}$, \\ Chia-Hao Chang ${ }^{1}$, Lih-Yu Chang ${ }^{1}$, Kai-Lun Yu ${ }^{1}$, Meng-Rui Lee ${ }^{1 *}$ and Jen-Chung Ko ${ }^{1}$
}

${ }^{1}$ Department of Internal Medicine, National Taiwan University Hospital Hsin-Chu Branch, Hsin-Chu, Taiwan, ${ }^{2}$ Department of Physical Medicine and Rehabilitation, National Taiwan University Hospital Hsin-Chu Branch, Hsin-Chu, Taiwan

Background: Comprehensive rehabilitation programs are recommended for patients with prolonged mechanical ventilation (PMV) to facilitate functional recovery and ventilator weaning, but whether the functional status after rehabilitation influences outcome has not been clearly evaluated. This study aimed to investigate the association between post-rehabilitation functional status and weaning and survival outcome in PMV patients.

Methods: We retrospectively enrolled PMV patients admitted to the respiratory care center (RCC), a post-ICU weaning facility with protocolized rehabilitation program, from January 2016 through December 2017. Functional status was measured by the de Morton Mobility Index (DEMMI), with a cut-off value set at 20 points. The primary outcomes were the weaning status at RCC discharge and hospital survival. The secondary outcomes were overall survival and survival at 3 months after RCC discharge. We followed patients until 3 months after RCC discharge or death. Logistic and Cox regressions were performed to identify significant parameters associated with weaning success and survival.

Results: In total, 320 patients were enrolled. The weaning success rate was $71.6 \%$. The survival rate at RCC discharge, hospital discharge, and 3 months after RCC discharge was $89.1,77.5$, and $66.6 \%$, respectively. Post-rehabilitation DEMMI $\geq$ 20 (odds ratio [OR], 3.514; 95\% confidence interval [Cl], 1.436-8.598; $P=0.006$ ) was the most significantly associated with weaning success. The weaning success and higher post-rehabilitation DEMMI were the two most significant independent factors associated with both hospital survival (weaning success, OR, 12.272; 95\% $\mathrm{Cl}$, 5.281-28.517; $P<0.001$; post-rehabilitation DEMMI $\geq 20$, OR, 6.298; 95\% Cl, 1.302-30.477; $P=0.022$ ) and survival at 3 months after RCC discharge (weaning success, OR, 38.788; 95\% Cl, 11.505-130.762; $P<0.001$; post-rehabilitation DEMMI $\geq 20$, OR, 4.830; 95\% Cl, 1.072-21.756; $P=0.040$ ). Post-rehabilitation $\mathrm{DEMMI} \geq 20$ remained significantly association with overall survival at 3 months after RCC discharge (hazard ratio, 0.237; 95\% Cl, 0.072-0.785; $P=0.018$ ). 
Conclusions: Post-rehabilitation functional status of PMV patients was independently associated with weaning success, as well as hospital and 3-month overall survival after RCC discharge. Post-rehabilitation, but not pre-rehabilitation, functional status was a significant parameter associated with weaning success and survival in patients requiring PMV.

Keywords: The de Morton Mobility Index, functional status, mortality, prolonged mechanical ventilation, rehabilitation, weaning

\section{INTRODUCTION}

One of the leading causes for intensive care unit (ICU) admission is acute respiratory failure with mechanical ventilator use (1). Due to the advancement in critical care medicine, many patients could survive through the acute stage of critical illness. About $10-20 \%$ of ICU patients, however, may require prolonged mechanical ventilation (PMV, defined as mechanical ventilator use for more than 21 days), and they consume about $37-50 \%$ of ICU resources (2). A recent systematic review showed that age, vasopressor use, thrombocytopenia, pre-existing renal disease, acute kidney injury or dialysis-dependent renal failure, and failure to wean from ventilator were strong prognostic factors for long-term mortality in patients requiring PMV (3). Among them, failure to wean has been reported as the most important prognostic factor (4).

Pulmonary rehabilitation with early mobilization and physiotherapy has been proposed as a component of multidisciplinary strategy to promote successful weaning in patients with PMV use (5). Several studies reported benefits of pulmonary rehabilitation in PMV patients including improvement in functional status (6-8), duration of spontaneous breathing trial (6), weaning rate (7), or even mortality $(7,8)$. Comprehensive rehabilitation programs are therefore recommended in chronic critically ill patients to speed up functional recovery and to reduce the risk of ventilator dependence or difficult weaning (9). Studies evaluating the association between functional status after routine pulmonary rehabilitation and weaning or survival outcome in patients receiving PMV, however, remain limited. Theoretically, the functional status after rehabilitation, a parameter of response to rehabilitation, should be a more reliable and proximate predictor for the weaning or survival outcome, than the acceptance of rehabilitation itself. Only one prospective study showed the change of functional status after rehabilitation was associated with weaning and survival rates in tracheostomized patients with PMV use (10). Whether post-rehabilitation functional status is associated with weaning and survival rates in all PMV patients regardless of the status with endotracheal intubation or tracheostomy remains unknown.

Abbreviations: APACHE, Acute Physiology and Chronic Health Evaluation; DEMMI, the de Morton Mobility Index; ICU, intensive care unit; $\mathrm{P}_{\text {Emax }}$, maximal expiratory pressure; $\mathrm{P}_{\mathrm{Imax}}$, maximal inspiratory pressure; PMV, prolonged mechanical ventilation; RCC, respiratory care center; RSBI, rapid shallow breathing index.
The aim of this study is to evaluate the association of functional status after pulmonary rehabilitation in PMV patients and the weaning and survival outcome. We hypothesize that functional status after pulmonary rehabilitation is associated better weaning and survival rates.

\section{METHODS}

\section{Settings and Population}

This study was conducted retrospectively in the 12-bed respiratory care center (RCC), a step-down and protocol-driven weaning facility, in the National Taiwan University Hospital Hsin-Chu branch, a university-affiliated hospital in northern Taiwan. PMV was defined as ventilator support for more than 21 days, according to the definition from the National Association for Medical Direction of Respiratory Care Consensus Conference in 2005 (11), and the regulations of the National Health Insurance in Taiwan. Patients anticipated to require PMV support were transferred to the RCC if they fulfilled the following criteria: (1) age $\geq 20$ years, (2) resolved acute critical illness without multiple organ failure, (3) fraction of inspiratory oxygen $\leq 0.40$ and positive end-expiratory pressure $\leq 8 \mathrm{~cm} \mathrm{H} \mathrm{H}_{2} \mathrm{O}$, and (4) stable hemodynamic conditions, with norepinephrine-equivalent dose $\leq 5 \mu \mathrm{g} / \mathrm{min}$. Patients who survived but failed ventilator weaning after a 6-week stay in the RCC were transferred to long-term care facilities. The nurse-topatient ratio in the RCC was 1:4, and one respiratory therapist is on duty during each shift. All patients transferred to the RCC received standardized respiratory therapist-driven protocol for ventilator weaning (Supplementary Figure 1). All eligible RCC patients finally requiring PMV were enrolled from January 2016 to December 2017. Only the last admission was included in the analysis if patients had been admitted to the RCC more than once during the study period. The Institutional Review Board (National Taiwan University Hospital Hsin-Chu Branch REC: 107-043-E) authority approved the study and waived the required informed consent.

\section{Rehabilitation Protocol and Physical Function Measurement}

All patients received the same physical training program conducted by experienced physical therapists 5 sessions per week during the entire RCC stay. The standardized session of physical training program included: (1) breathing exercise (deep [with doubling tidal volume] and segmental) in semi-recumbent position $\left(\geq 45^{\circ}\right)$, followed by cough training, with 10 repetitions 
per set for 1-2 sets; (2) chest mobility exercise, containing manual technique, and arm elevation with upper trunk lateral flexion, with 10 repetitions; (3) strengthening exercise for respiratory, abdominal and upper and lower limb muscles, containing antigravity elevation and resistance band exercise (for upper limbs), or straight leg raising or bridging exercise (for lower limbs), with 10 repetitions per set for 2-3 sets; (4) range-ofmotion exercises (active, passive, or active-assisted, according to individual tolerance) of upper and lower extremities, with 10 repetitions; (5) functional activity training (bed mobility such as rolling or bridging, sitting, standing, or walking with adequate ventilator and oxygenation support), with 1-2 repetitions; and (6) endurance training, containing sitting/standing for $10 \mathrm{~min}$, or limbs exercise/biking for $5 \mathrm{~min}$. Each session lasted for 15$50 \mathrm{~min}$ (as long as tolerated), with all 6 elements performed as much as possible, according to individual tolerance and program progression. In neurologically impaired patients that could not follow orders, the endurance training was not performed.

Functional status was measured by the de Morton Mobility Index (DEMMI), originally developed to assess the mobility status in hospitalized elderly patients (12). It was later found to be feasible, reliable and valid in the ICU setting (13). The raw DEMMI, ranging from 0 to 19 points, was a 15-item unidimensional measure of mobility. The ordinal scale was then Rasch converted to a 0 to 100 interval scale, with a score of 0 and 100 indicating no and full mobility, respectively. The DEMMI was measured twice in the RCC by an experience physical therapist, at RCC admission (pre-rehabilitation) and before RCC discharge (post-rehabilitation), respectively. If extubation was performed during RCC stay, the measurement of postrehabilitation DEMMI was done just before extubation.

DEMMI cut-off level for analysis in our study was determined according to the following steps and rationale. First, the corresponding optimal cut-off values determined by Youden index point on the receiver operating characteristic (ROC) curves of post-rehabilitation DEMMI were 17.5 (area under curve [AUC], 0.547; 95\% confidence interval [CI], 0.479-0.615), 11.5 (AUC, 0.638; 95\% CI, 0.574-0.702), and 11.5 (AUC, 0.664; 95\% CI, 0.604-0.725) for weaning success, hospital survival, and 3-month survival after RCC discharge, respectively. Also, the reported minimal clinically important difference for DEMMI ranged from 10 to 20 points (14-16). The cut-off value of DEMMI in this study was therefore set at 20 points, which was the lowest converted DEMMI score more than or equal to the above mentioned cut-off values and minimal clinically important differences for consistency and simplicity.

\section{Data Collection}

All clinical information including survival data were obtained from patient medical records and respiratory care database. The retrieved patient information during ICU stay included baseline demographic and cormorbidities, medical or surgical type of ICU admission, cause of respiratory failure, septic shock (17), and acute respiratory distress syndrome (18) in ICU. The Acute Physiology and Chronic Health Evaluation (APACHE) II was determined at ICU admission and at the time of RCC transfer, as an assessment of the disease severity (19). The retrieved patient information in RCC included the presence of tracheostomy, body mass index, Glasgow coma scale, and the results of various laboratory tests. The weaning parameters, which included maximal inspiratory pressure $\left(\mathrm{P}_{\operatorname{Imax}}\right)$, maximal expiratory pressure $\left(\mathrm{P}_{\mathrm{Emax}}\right)$, rapid shallow breathing index (RSBI), tidal volume, and minute ventilation, were measured twice, at RCC admission (prerehabilitation) and before extubation or RCC discharge (postrehabilitation), respectively. The established cut-off values for weaning evaluation were used as references, including $\mathrm{P}_{\text {Imax }}$ : $-20 \mathrm{~cm} \mathrm{H} \mathrm{H}_{2} \mathrm{O}, \mathrm{P}_{\text {Emax }}$ : $+30 \mathrm{~cm} \mathrm{H}_{2} \mathrm{O}$, RSBI: 105, tidal volume: 5 $\mathrm{mL} / \mathrm{kg}$, and minute ventilation, $10 \mathrm{~L} / \mathrm{min}$ (20). $\mathrm{P}_{\operatorname{Imax}}$ and $\mathrm{P}_{\mathrm{Emax}}$ were measured by a conventional pressure gauge connected to a one-way valve for airway occlusion while the patients were placed in sitting position with temporary ventilator disconnection. For $\mathrm{P}_{\text {Imax }}$ measurement, the patients exhaled as far as possible and then inhaled as forcefully as possible with the pressure level maintained for at least $2 \mathrm{~s}$. The patients inhaled/exhaled conversely for $\mathrm{P}_{\mathrm{Emax}}$ measurement. Up to 5 consecutive efforts were performed and the highest value was recorded (21). We followed all the patients until 3 months after RCC discharge or death.

\section{Outcome Measurements}

The primary outcome measurements were the weaning status (success or failure) at RCC discharge and survival status at hospital discharge. The secondary outcome measurements were survival at 3 months after RCC discharge and overall survival after respiratory failure. Weaning success was defined as liberation from the ventilator (both invasive and non-invasive) for more than 5 days, according to the prospective payment system of ventilator dependents' managed care by the National Health Insurance in Taiwan (22). Patients were classified as weaning failure if they failed mechanical ventilator liberation at RCC discharge, returned to the ICU for deteriorated critical illness, or died during the RCC stay.

\section{Statistical Analysis}

Data are expressed as the mean \pm standard deviation and number (\%) for continuous and categorical variables, respectively. Continuous variables were compared using Student's $t$-test, and categorical variables were compared using Pearson's $\chi 2$ or Fisher's exact test, as appropriate. Multivariate logistic regression models were used to identify significant clinical factors associated with weaning success, hospital survival, and 3-month survival outcome in the study population. KaplanMeier curves were plotted for overall survival after respiratory failure, and the differences between patient subgroups were compared using the log-rank test. Multivariate Cox proportionalhazards model with adjustment for variables with statistical significance in the univariate analysis to identify significant clinical factors associated with overall survival after respiratory failure. Variables included in the logistic and Cox multivariate analysis were statistically significant factors in the univariate analysis. Both logistic and Cox regression analyses were 
TABLE 1 | Clinical characteristics at baseline and intensive care unit admission.

\begin{tabular}{|c|c|c|c|c|c|c|c|}
\hline \multirow[b]{3}{*}{$N$} & \multicolumn{2}{|c|}{ Entire population } & \multicolumn{4}{|c|}{ Weaning outcome } & \multirow[t]{3}{*}{$P$} \\
\hline & & & \multicolumn{2}{|c|}{ Success } & \multicolumn{2}{|c|}{ Failure } & \\
\hline & 320 & & 229 & & 91 & & \\
\hline Age (years) & $70.9 \pm 13.9$ & & $70.4 \pm 13.9$ & & $72.1 \pm 13.9$ & & 0.324 \\
\hline Male sex & 202 & $(63.1)$ & 142 & $(62.0)$ & 60 & $(65.9)$ & 0.511 \\
\hline \multicolumn{8}{|l|}{ Co-morbidities } \\
\hline Coronary artery disease & 69 & $(21.6)$ & 51 & $(22.3)$ & 18 & $(19.8)$ & 0.625 \\
\hline Congestive heart failure & 67 & (20.9) & 45 & $(19.7)$ & 22 & $(24.2)$ & 0.369 \\
\hline Chronic obstructive pulmonary disease & 63 & $(19.7)$ & 35 & $(15.3)$ & 28 & $(30.8)$ & 0.002 \\
\hline Other chronic lung disease & 37 & $(11.6)$ & 20 & $(8.7)$ & 17 & $(18.7)$ & 0.012 \\
\hline Diabetes mellitus & 144 & $(45.0)$ & 104 & $(45.4)$ & 40 & $(44.0)$ & 0.813 \\
\hline Cirrhosis & 14 & $(4.4)$ & 8 & $(3.5)$ & 6 & $(6.6)$ & 0.234 \\
\hline Chronic kidney disease & 84 & (26.3) & 54 & $(23.6)$ & 30 & $(33.0)$ & 0.085 \\
\hline End-stage renal disease & 28 & $(8.8)$ & 18 & $(7.9)$ & 10 & $(11.0)$ & 0.372 \\
\hline Old stroke & 66 & $(20.6)$ & 53 & $(23.1)$ & 13 & $(14.3)$ & 0.077 \\
\hline Other neurologic disease & 47 & $(14.7)$ & 31 & $(13.5)$ & 16 & $(17.6)$ & 0.356 \\
\hline Cancer & 48 & $(15.0)$ & 30 & $(13.1)$ & 18 & $(19.8)$ & 0.131 \\
\hline Cause of respiratory failure & & & & & & & $<0.001$ \\
\hline Pulmonary & 129 & $(40.3)$ & 81 & $(35.4)$ & 48 & $(52.7)$ & \\
\hline Cardiovascular & 28 & $(8.8)$ & 18 & $(7.9)$ & 10 & $(11.0)$ & \\
\hline Neurologic & 35 & $(10.9)$ & 31 & $(13.5)$ & 4 & $(4.4)$ & \\
\hline Post-operative & 83 & (25.9) & 71 & $(31.0)$ & 12 & $(13.2)$ & \\
\hline Others & 45 & $(14.1)$ & 28 & $(12.2)$ & 17 & $(18.7)$ & \\
\hline \multicolumn{8}{|l|}{ ICU admission } \\
\hline APACHE II & $20.2 \pm 7.1$ & & $19.6 \pm 6.7$ & & $21.9 \pm 7.9$ & & 0.025 \\
\hline Septic shock & 107 & (33.4) & 68 & $(29.7)$ & 39 & $(42.9)$ & 0.024 \\
\hline ARDS & 15 & $(4.7)$ & 9 & (3.9) & 6 & $(6.6)$ & 0.379 \\
\hline
\end{tabular}

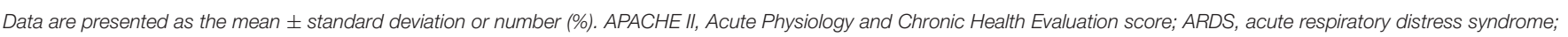
ICU, intensive care unit.

performed with backward selection using the entry and stay criteria at 0.05 and 0.1 , respectively. We used stepwise regression in order to manage large amount of potential predictor variables and to choose the best predictor variables from the available options. A two-sided $P$-value $<0.05$ was considered statistically significant. All analyses were performed using SPSS version 18.0 for Windows (IBM Corporation, Armonk, NY, USA).

\section{RESULTS}

\section{Study Population}

Between January 2016 and December 2017, 2,653 patients were admitted to our ICUs with mechanical ventilation support. Among them, 320 patients requiring PMV support were subsequently transferred to the RCC. The mean age was 70.9 years and $202(63.1 \%)$ were men. Pulmonary disease $(40.3 \%)$ and post-operative care $(25.9 \%)$ were the two most common causes of respiratory failure. The baseline characteristics and demographic features of the study population were shown in Table 1. The overall weaning success rate at RCC discharge was $71.6 \%(229 / 320)$. The survival rate at RCC discharge, hospital discharge, and 3 months after RCC discharge was $89.1 \%(285 / 320), 77.5 \%$ (248/320), and 66.6\% (201/302), respectively. The flow diagram of the study protocol was shown in Figure 1.

\section{Significant Clinical Factors Associated With Weaning Success}

The clinical characteristics of patients with weaning success and those with weaning failure were described in Tables 1, 2. Compared to the PMV patients with weaning failure, those with weaning success had fewer pulmonary co-rmorbidites, fewer pulmonary causes and more post-operative causes of respiratory failure, lower APACHE II score both at ICU admission and RCC transfer, a lower incidence of septic shock at ICU admission, lower creatinine level and higher platelet, hemoglobin, and albumin level at RCC transfer. While the proportion of patients with $\mathrm{DEMMI} \geq 20$ before rehabilitation did not significantly differ between these 2 groups, patients with weaning success were more likely to have post-rehabilitation DEMMI $\geq 20$ than those with weaning failure (26.6 vs. $15.4 \%, P=0.032$ ). Similarly, while all the pre-rehabilitation weaning parameters did not differ between these 2 groups, patients with weaning 


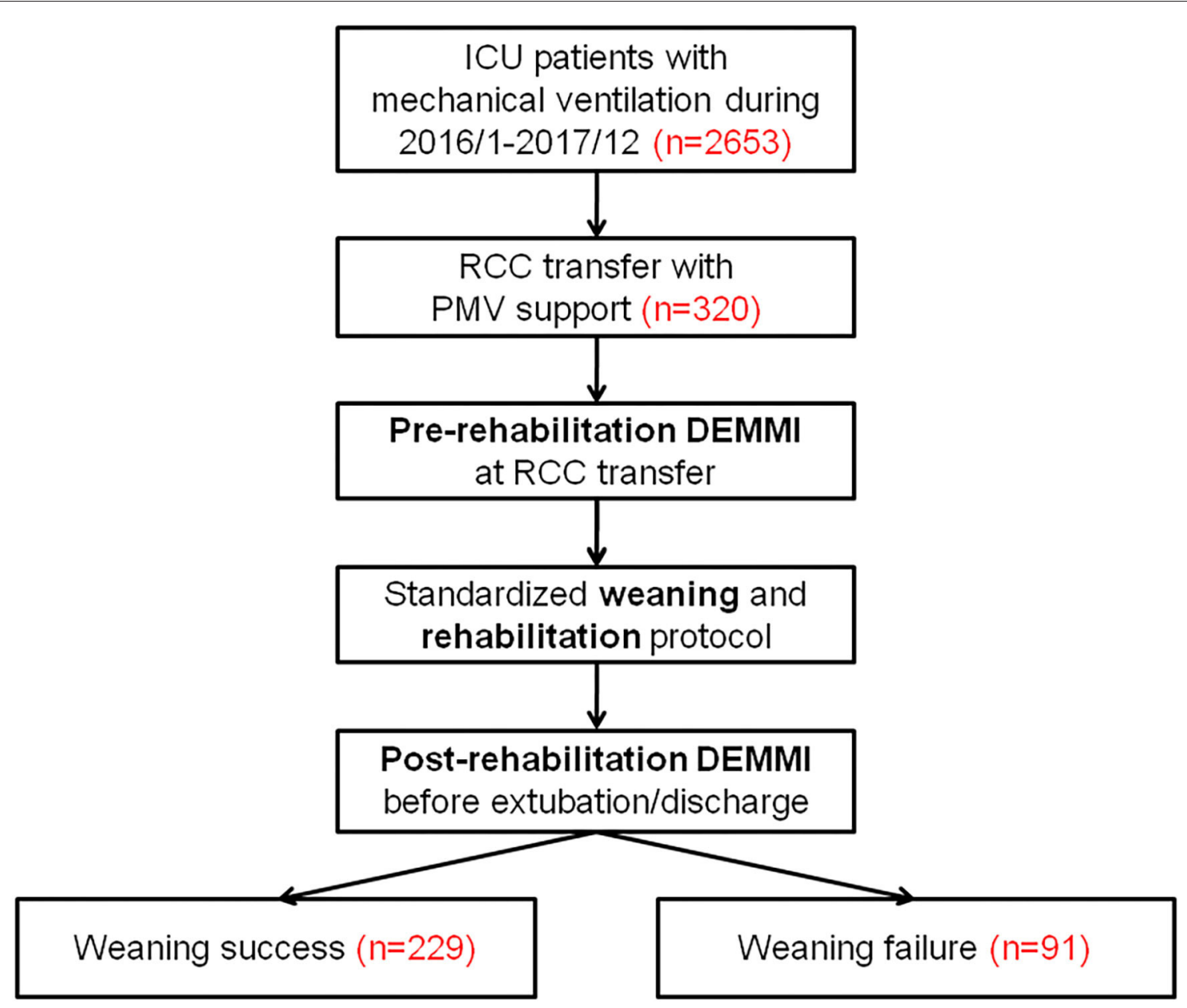

FIGURE 1 | The flow diagram of the study protocol. DEMMI, the de Morton Mobility Index; ICU, intensive care unit; PMV, prolonged mechanical ventilation; RCC, respiratory care center.

success had higher post-rehabilitation $\mathrm{P}_{\mathrm{Emax}}$ (66.8 vs. $46.6 \%, P$ $=0.002$ ) and lower post-rehabilitation RSBI (76.8 vs. $63.0 \%$, $P=0.024)$ than those with weaning failure. The duration of rehabilitation did not significantly differ between patients with weaning success and those with weaning failure (11.6 \pm 4.9 days vs. $13.0 \pm 7.8$ days; $P=0.095$ ), or between groups with postrehabilitation DEMMI $\geq 20$ and those with post-rehabilitation DEMMI $<20(12.4 \pm 5.6$ days vs. $11.9 \pm 6.0$ days; $P=$ $0.500)$. Multivariate logistic regression models showed that the absence of chronic obstructive pulmonary disease (odds ratio [OR], 0.298; 95\% confidence interval [CI], 0.120-0.742; $P=$ 0.009 ), post-operative respiratory failure (OR, 2.450; 95\% CI, 1.002-5.992; $P=0.0496)$, post-rehabilitation DEMMI $\geq 20$ (OR, 3.514; 95\% CI, 1.436-8.598; $P=0.006$ ), post-rehabilitation RSBI $\leq 105$ (OR, 2.644; 95\% CI, 1.290-5.419; $P=0.008$ ), and higher platelet counts (increments of $10^{4} / \mu \mathrm{L}$; OR, 1.031; 95\% CI, 1.0051.058; $P=0.020$ ) were significantly associated with weaning success (Table 3 ).

\section{Significant Clinical Factors Associated With Hospital Survival and 3-Month Survival After RCC Discharge}

The clinical characteristics of survivors and non-survivors at hospital discharge or 3 months after RCC discharge in PMV patients are shown in Supplementary Tables 1, 2. Among 320 PMV patients, 302 (94.4\%) had follow-up 3-month survival data. In detail, among $248 \mathrm{PMV}$ patients who survived at hospital discharge, 201 (81.0\%) survived and 29 (11.7\%) died at 3 month after RCC discharge respectively, while the survival status was unavailable in the remaining 18 patients $(7.3 \%)$. While the proportion of patients with DEMMI $\geq 20$ before rehabilitation did not significantly differ between groups with survival and mortality at hospital discharge or 3 month after RCC discharge, the PMV survivors at hospital discharge or 3 month after RCC discharge were more likely to have post-rehabilitation DEMMI $\geq 20$ than non-survivors (hospital discharge: 29.0 vs. $4.2 \%, P<0.001 ; 3$ months after RCC 
TABLE 2 | Clinical characteristics during respiratory care center hospitalization.

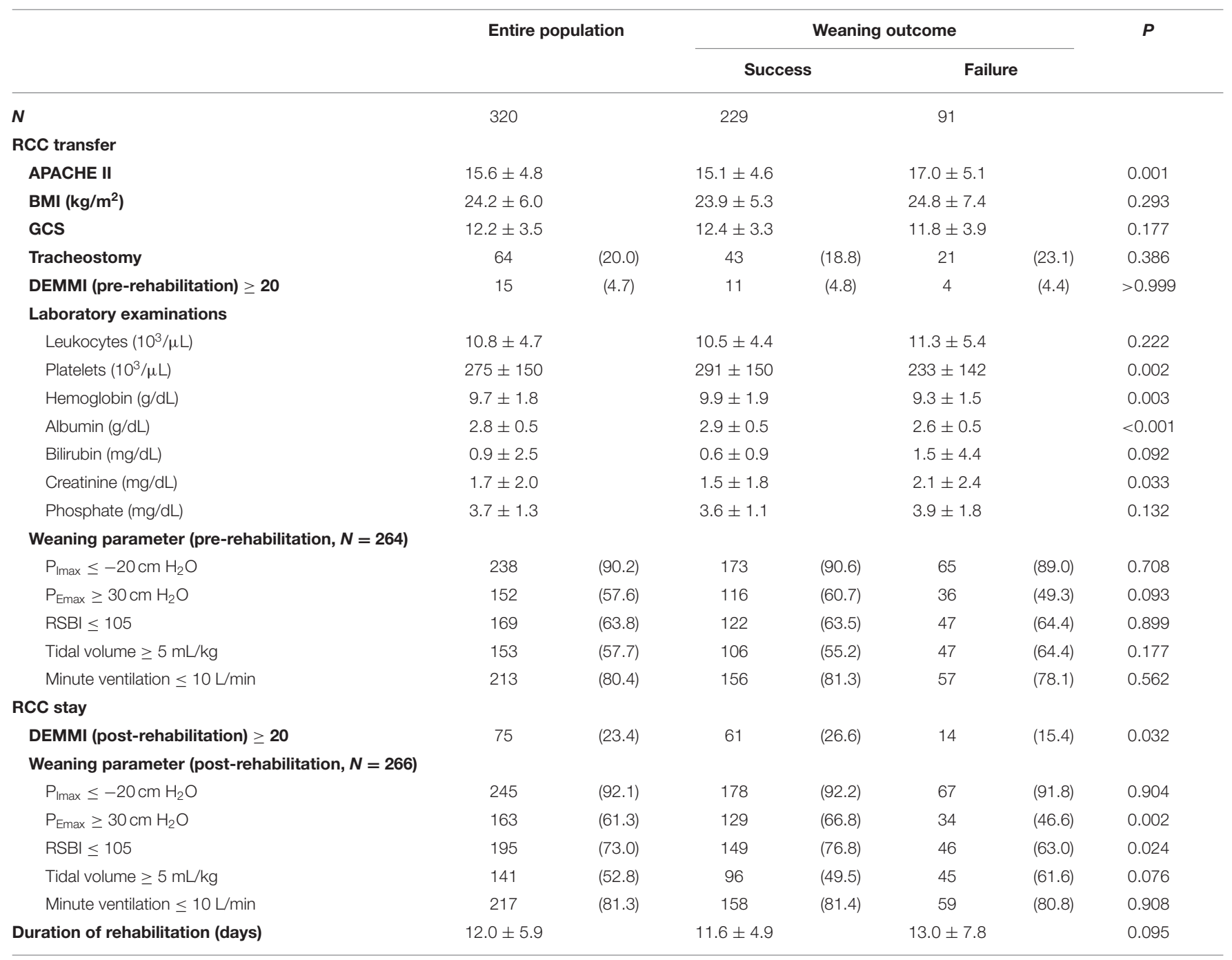

Data are presented as mean \pm standard deviation or number (\%). APACHE II, Acute Physiology and Chronic Health Evaluation score; BMI, body mass index; DEMMI, the de Morton Mobility Index; GCS, Glasgow Coma Scale; PEmax, maximal expiratory pressure; PImax, maximal inspiratory pressure; RCC, respiratory care center; RSBI, rapid shallow breath index.

discharge: 33.3 vs. $5.9 \%, P<0.001)$. Similarly, while all the pre-rehabilitation weaning parameters did not differ between survivors and non-survivors at hospital discharge or 3 months after RCC discharge, both of those survivors had higher postrehabilitation $P_{\text {Emax }}$ than those non-survivors. The duration of rehabilitation did not significantly differ between patients with hospital survival and those with hospital mortality (12.2 \pm 5.7 days vs. $11.2 \pm 6.4$ days; $P=0.203$ ), or between groups with survival and mortality at 3 month after RCC discharge (12.1 \pm 5.9 days vs. $11.6 \pm 6.2$ days; $P=0.476)$. Multivariate logistic regression models showed that weaning success, postrehabilitation DEMMI $\geq 20$, and platelets were significantly associated with both hospital survival (weaning success: OR, 12.272; 95\% CI, 5.281-28.517; $P<0.001$; post-rehabilitation DEMMI: OR, 6.298; 95\% CI, 1.302-30.477; $P=0.022$; platelets: increments of $10^{4} / \mu \mathrm{L}$; OR, 1.045; 95\% CI, 1.010-1.082; $P=$ 0.012 ) and 3-month survival after RCC discharge (weaning success: OR, 38.788; 95\% CI, 11.505-130.762; $P<0.001$; postrehabilitation DEMMI: OR, 4.830; 95\% CI, 1.072-21.756; $P=$ 0.040 ; platelets: increments of $10^{4} / \mu \mathrm{L}$; OR, 1.066 ; $95 \%$ CI, $1.018-$ $1.117 ; P=0.007)($ Table 4).

\section{Association Between Different Pre-rehabilitation and Post-rehabilitation DEMMI and Weaning and Survival Status}

To further evaluate the association between various postrehabilitation DEMMI and survival status at different time points, we divided the study population into 4 groups according to post-rehabilitation DEMMI: 0-9, 10-19, 20-29, and 30-39. 
TABLE 3 | Multivariate logistic regression models for significant clinical characteristics associated with weaning success at RCC discharge*.

\begin{tabular}{|c|c|c|c|c|c|}
\hline Parameters & $\beta$ & SE & Odds ratio & $(95 \% \mathrm{Cl})$ & $\boldsymbol{P}$ \\
\hline COPD (yes vs. no) & -1.210 & 0.465 & 0.298 & $(0.120-0.742)$ & 0.009 \\
\hline \multicolumn{6}{|l|}{ Cause of respiratory failure } \\
\hline Pulmonary & & & & 1 & - \\
\hline Cardiovascular & -0.587 & 0.055 & 0.556 & $(0.187-1.650)$ & 0.290 \\
\hline Neurologic & 0.460 & 0.710 & 1.584 & (0.394-6.363) & 0.517 \\
\hline DEMMI (post-rehabilitation, $\geq 20$ vs. $<20$ ) & 1.257 & 0.457 & 3.514 & $(1.436-8.598)$ & 0.006 \\
\hline RSBI (post-rehabilitation, $\leq 105$ vs. $>105$ ) & 0.972 & 0.366 & 2.644 & $(1.290-5.419)$ & 0.008 \\
\hline Platelets $\left(10^{4} / \mu \mathrm{L}\right)$ & 0.031 & 0.013 & 1.031 & $(1.005-1.058)$ & 0.020 \\
\hline Creatinine (mg/dL) & -0.142 & 0.075 & 0.868 & $(0.749-1.006)$ & 0.060 \\
\hline
\end{tabular}

Cl, confidence interval; COPD, chronic obstructive pulmonary disease; DEMMI, the de Morton Mobility Index; RCC, respiratory care center; RSBI, rapid shallow breath index; SE, standard error

${ }^{*}$ Variables with statistical significance $(P<0.05)$ in the univariate analyses (Tables 1, 2) were included in the multivariate logistic regression models. Backward variable selection was performed, and the criteria of P-values for entry and stay were set at 0.05 and 0.10 , respectively. Details of the regression model establishment were shown in Supplementary Table 5 .

TABLE 4 | Multivariate logistic regression models for significant clinical characteristics associated with hospital survival and 3-month survival after RCC discharge*.

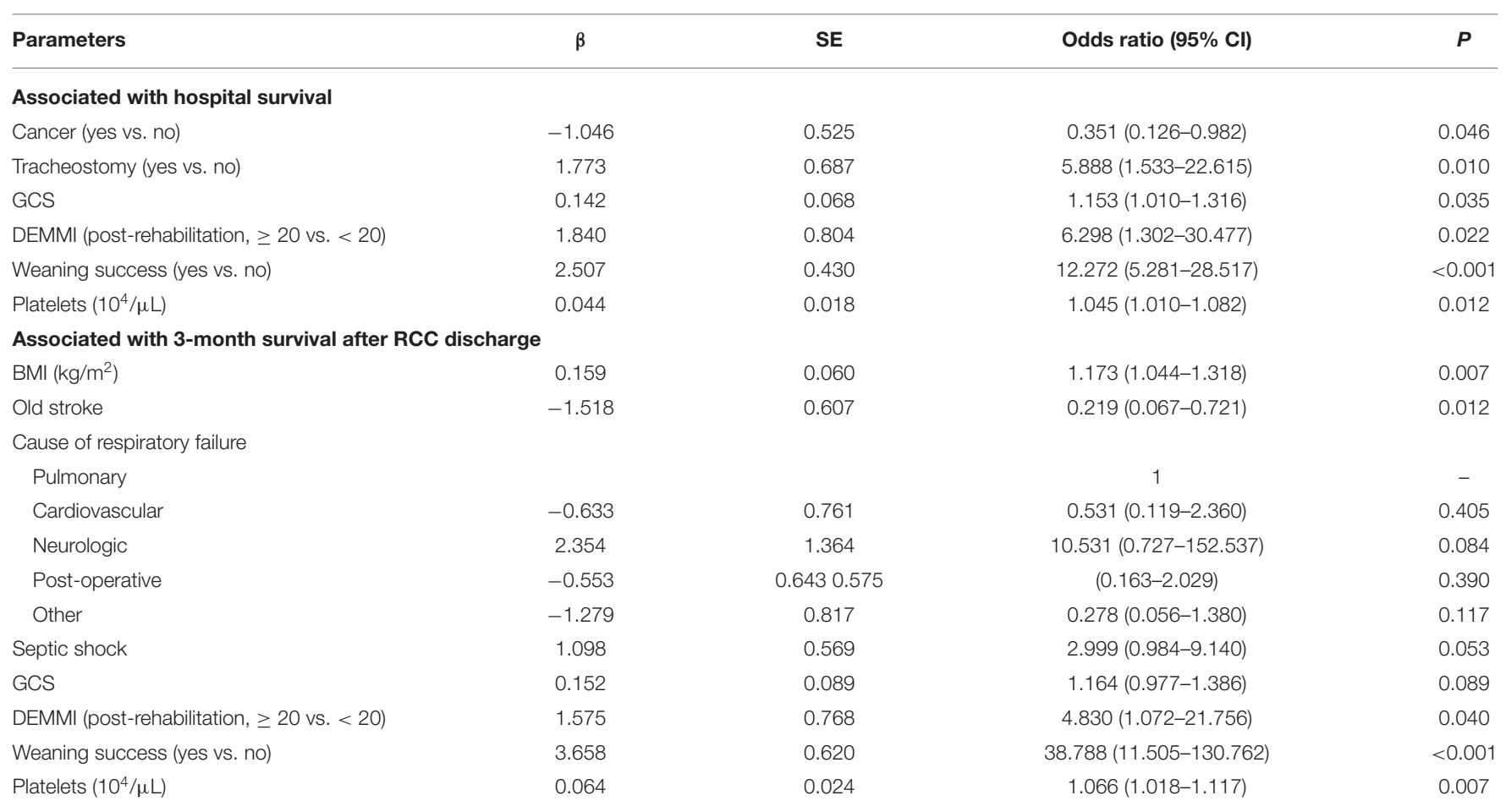

BMI, body mass index; Cl, confidence interval; DEMMI, the de Morton Mobility Index; GCS, Glasgow Coma Scale; RCC, respiratory care center; SE, standard error. *Variables with statistical significance $(P<0.05)$ in the univariate analyses (Supplementary Tables 1,2) were included in the multivariate logistic regression models. Backward variable selection was performed, and the criteria of P-values for entry and stay were set at 0.05 and 0.10 , respectively. Details of the regression model establishment were shown in Supplementary Tables 6, 7.

The PMV patients with higher post-rehabilitation DEMMI had higher survival rate at RCC discharge, hospital discharge, and 3 months after RCC discharge (all $P<0.001$ ). The 3-month survival rate after RCC discharge surpassed 90 and $95 \%$ in patients with post-rehabilitation DEMMI $\geq 20$ and $\geq 30$, respectively (Figure 2 ).

We also compared different weaning and survival status among patient groups stratified by different pre- and 


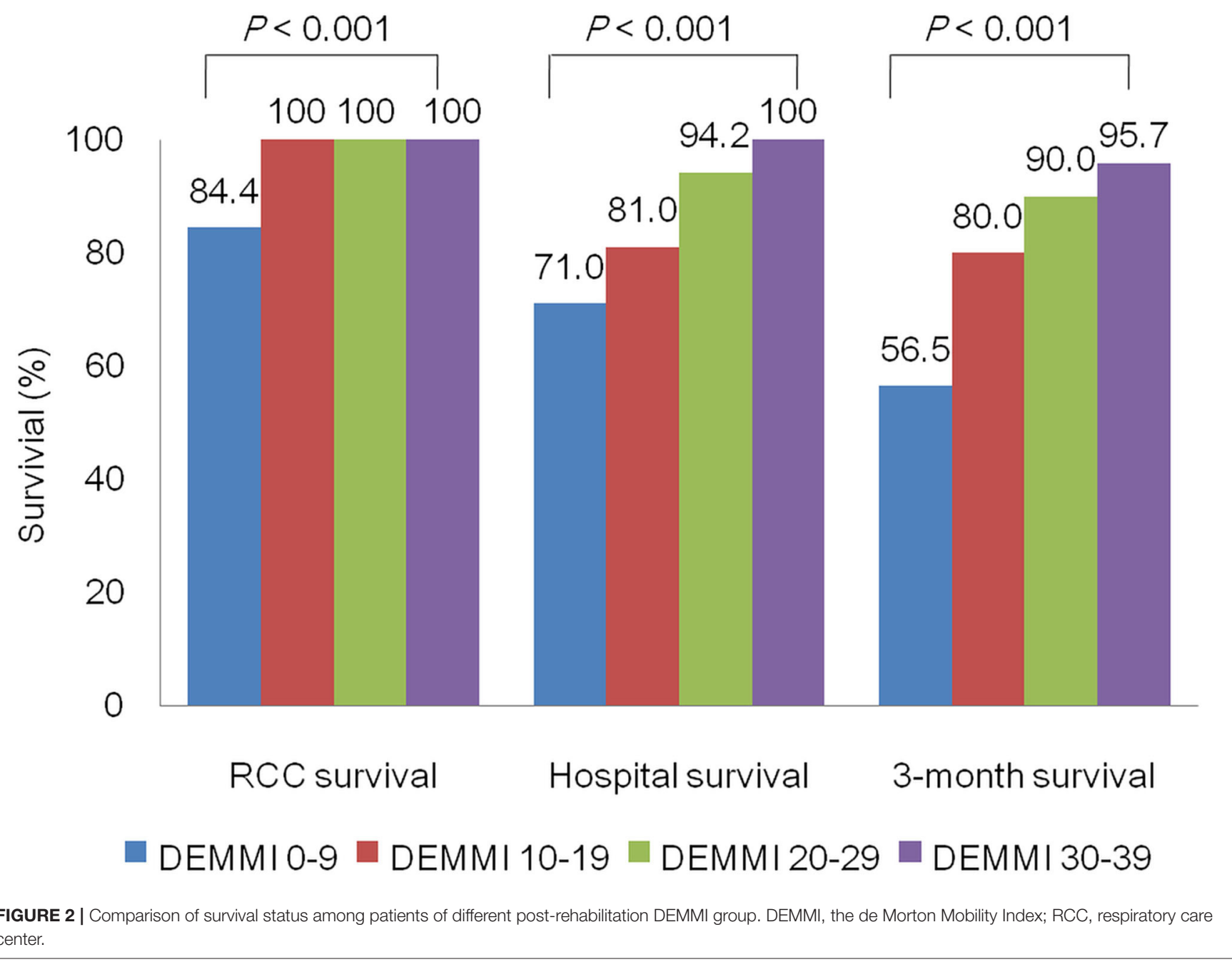

post-rehabilitation DEMMI combination, as shown in Figure 3. All patients with pre-rehabilitation DEMMI $\geq 20$ had post-rehabilitation DEMMI $\geq 20$. Patients with postrehabilitation DEMMI $\geq 20$ had similar weaning and survival outcome, regardless of their corresponding pre-rehabilitation DEMMI. In the contrary, in patients with pre-rehabilitation DEMMI $<20$, those who had post-rehabilitation DEMMI $\geq 20$ (rehabilitation responders) had significantly higher weaning ( 83.3 vs. $68.6 \%, P=0.023)$ and survival (100 vs. $85.7 \%, P=0.002$, at RCC discharge; 96.7 vs. $71.8 \%, P<$ 0.001 , at hospital discharge; 93.2 vs. $58.5 \%, P<0.001$, at 3 months after RCC discharge) rate at various time points than those who still had post-rehabilitation DEMMI $<20$ (rehabilitation non-responders).

Among patients with weaning failure, patients with postrehabilitation DEMMI < 20 (respiratory care ward transfer: $30.8 \%$ [28/77]; ICU transfer: $19.8 \%$ [18/77]; death in RCC: $34.1 \%$ [31/77]) were more likely to end up with ICU transfer or death in RCC than those with post-rehabilitation DEMMI $\geq 20$ (respiratory care ward transfer: $15.4 \%$ [14/14]; ICU transfer or death in RCC: $0 \%[0 / 14])(P<0.001)$.

\section{Overall Survival in Patients With Different Post-rehabilitation DEMMI}

Kaplan-Meier survival curves were plotted for the different patient subgroups (Figure 4). Patients with post-rehabilitation DEMMI $\geq 20$ had significantly longer overall survival than those with post-rehabilitation DEMMI $<20$ (median survival: $>150$ days, not reached vs. 155 days, $P<0.001$ ). The duration of rehabilitation was not significantly associated with overall survival. A multivariate Cox proportional hazards model with adjusting for significant covariates showed that weaning success (hazard ratio [HR], 0.168; 95\% CI, 0.096-0.293; $P<0.001$ ), postrehabilitation DEMMI $\geq 20$ ( HR, 0.237; 95\% CI, 0.072-0.785; $P=0.018$ ), and higher platelet counts (increments of $10^{4} / \mu \mathrm{L}$; HR, 0.974; 95\% CI, 0.953-0.995; $P=0.017$ ) were significantly associated with better overall survival, while higher total bilirubin 


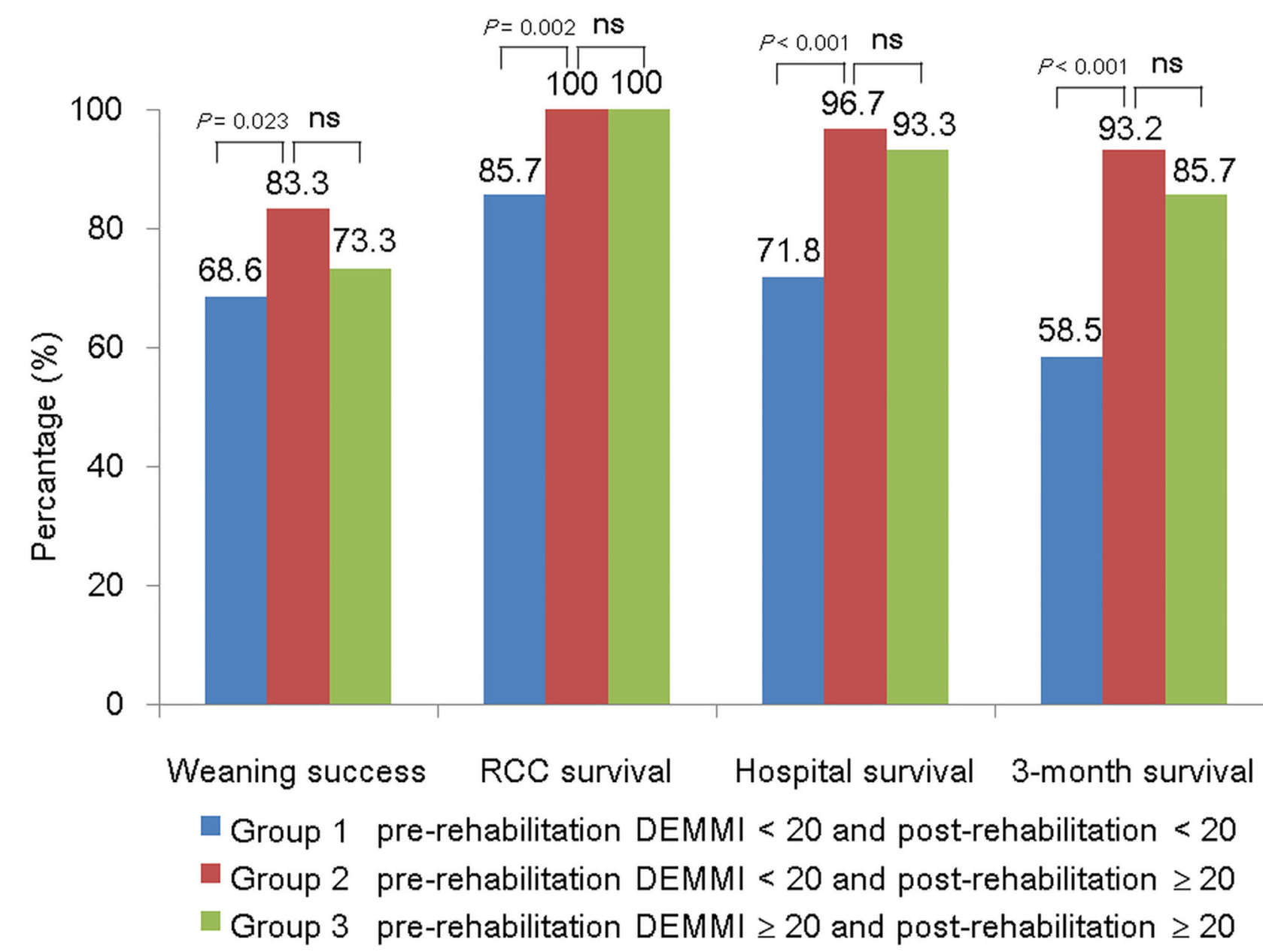

FIGURE 3 | Comparison of weaning and survival status among patient groups stratified by different pre- and post-rehabilitation DEMMI combination. DEMMI, the de Morton Mobility Index; ns, not significant; RCC, respiratory care center.

level (HR, 1.062; 95\% CI, 1.008-1.119; $P=0.025$ ), the presence of end-stage renal disease (HR, 2.368; 95\% CI, 1.034-5.420; $P=$ $0.041)$ and old stroke (HR, 1.974; 95\% CI, 1.047-3.723; $P=0.036)$ were associated with worse overall survival (Table 5).

\section{DISCUSSION}

Our study revealed that, in patient with PMV use, postrehabilitation DEMMI was an important prognostic factor independently associated with weaning success, hospital survival and 3-month survival after RCC discharge. Post-rehabilitation DEMMI, but not pre-rehabilitation DEMMI or the change of DEMMI after rehabilitation, could predict weaning success and short-term and mid-term survival in patients requiring PMV.

DEMMI as a functional measurement tool has been validated in inpatient population (15) and ICU population (13). Using DEMMI as functional status measurement in the post-ICU rehabilitation cohort has not yet been evaluated. In this study, post-rehabilitation functional status in PMV patients, as measured using DEMMI, showed significant correlation with weaning success, hospital survival and 3-months survival after RCC discharge. Our study showed that DEMMI could be used for functional status evaluation in post-ICU population requiring PMV and also an important prognostic factor for weaning and survival outcome.

Although previous studies evaluating the effectiveness of pulmonary rehabilitation programs on weaning rate in patients with PMV use showed conflicting results $(6-8,23)$, routine pulmonary rehabilitation had been adopted as a facet of multidisciplinary care for PMV patients to facilitate weaning. In this study, all enrolled PMV received standardized pulmonary rehabilitation protocol, but only those patients with higher postrehabilitation functional status had significant higher weaning rate. Functional response after pulmonary rehabilitation, therefore, may be more important than receiving pulmonary rehabilitation itself on weaning outcome in patients requiring PMV. In patients with PMV use, underlying pulmonary derangements such as the absence of chronic obstructive 


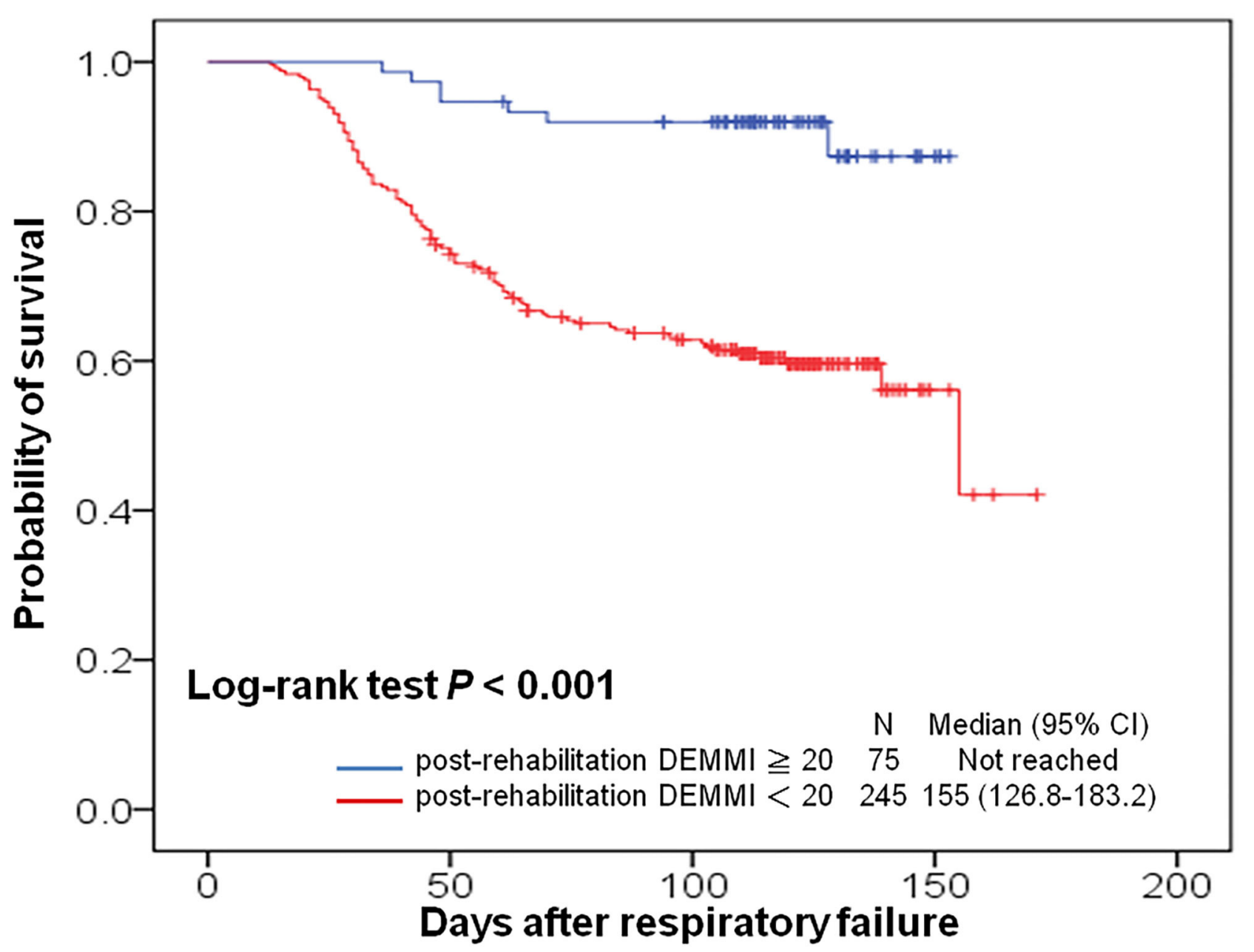

FIGURE 4 | Kaplan-Meier curves for overall survival in patients with prolonged mechanical ventilation, with stratification by post-rehabilitation DEMMI. Cl, confidence interval; DEMMI, the de Morton Mobility Index.

pulmonary disease and the presence of post-operative cause of respiratory failure were independently associated with weaning success. But the post-rehabilitation functional status was the most important determinant independently associated with weaning success, emphasizing the significance of pulmonary rehabilitation during RCC stay.

A recent meta-analysis showed age, thrombocytopenia, vasopressor requirement, failed ventilator disconnection, preexisting kidney disease, and acute kidney injury or hemodialysis requirement as 6 important prognostic factors for long-term mortality in PMV patients (3). In another prospective observational study, weaning outcome was the most important prognostic factor for 1-year mortality in patients requiring PMV (4), which remained significant even in cancer patients with PMV use (24). In our study, the finding that weaning outcome was the most important prognostic factor for both hospital survival and 3-month survival after RCC discharge is in general agreement with previous existing literatures. Our finding that post-rehabilitation functional status is also an important prognostic factor for both hospital survival and 3-month survival after RCC discharge was rarely reported in the literature (25). Even in patients with weaning failure, all patients with post-rehabilitation DEMMI $\geq 20$ were transferred alive to respiratory care wards without death or ICU transfer. Since post-rehabilitation functional status was also an independent factor for weaning success which itself had been shown to be the most important factor for mortality, the influence of post-rehabilitation functional status on survival outcome may extend beyond weaning.

In this study, several confounders and biases may exist and we have adjusted it in our final model. For instance, time of hospitalization in rehabilitation may lead to bias related to the time-dependent association. The duration of receiving rehabilitation which roughly equaled to duration of RCC stay, however, was not significantly different in patients with different outcomes regarding weaning, hospital survival, 3-month survival 
TABLE 5 | Multivariate Cox regression models for significant clinical characteristics associated with overall survival after respiratory failure*.

\begin{tabular}{lccc}
\hline Parameters & Hazard ratio & $\mathbf{( 9 5 \% ~ C l )}$ & $\boldsymbol{P}$ \\
\hline Age (years) & 1.021 & $(0.998-1.045)$ & 0.080 \\
$\begin{array}{l}\text { End-stage renal disease (yes } \\
\text { vs. no) }\end{array}$ & 2.368 & $(1.034-5.420)$ & 0.041 \\
Old stroke (yes vs. no) & 1.974 & $(1.047-3.723)$ & 0.036 \\
GCS & 0.929 & $(0.856-1.008)$ & 0.076 \\
Platelets (104/ $\mu$ L) & 0.974 & $(0.953-0.995)$ & 0.017 \\
Bilirubin (mg/dL) & 1.062 & $(1.008-1.119)$ & 0.025 \\
DEMMl (post-rehabilitation, $\geq$ & 0.237 & $(0.072-0.785)$ & 0.018 \\
20 vs. $<20)$ & & & \\
Weaning success (yes vs. no) & 0.168 & $(0.096-0.293)$ & $<0.001$ \\
\hline
\end{tabular}

Cl, confidence interval; DEMMI, the de Morton Mobility Index; GCS, Glasgow Coma Scale. *Variables with statistical significance $(P<0.05)$ in the univariate analyses (Supplementary Table 3) were included in the multivariate Cox regression models. Backward variable selection was performed, and the criteria of P-values for entry and stay were set at 0.05 and 0.10 , respectively. Details of the regression model establishment were shown in Supplementary Table 8.

after RCC discharge, or overall survival. The rehabilitation duration was also similar in patients with different postrehabilitation DEMMI status. Post-rehabilitation DEMMI $\geq 20$ remained significantly association with weaning success, hospital survival, and 3-month survival after RCC discharge in the multivariate logistic regression analysis, as well as overall survival in the multivariate Cox regression analysis. The benefit of longer rehabilitation time may be offset by the underlying physiological derangement causing prolonged RCC stay. Furthermore, the frequency of DEMMI evaluation may also be a potential confounder. In our study, DEMMI was universally examined twice for all patients and this ensured that the frequency of DEMMI was similar among all patients. Understanding the functional status of PMV patients more frequently may allow physicians and physical therapists to timely adjust treatment and rehabilitation programs, and therefore improve weaning and survival outcome. The role of evaluating DEMMI may extend from a prognostic factor to guidance for individualized rehabilitation program.

A previous retrospective study showed poor functional status at admission to post-ICU acute care hospital was significantly associated with higher 1-year mortality (26). In our study, the pre-rehabilitation baseline functional status was not associated with weaning or survival outcome. In contrast, only higher postrehabilitation functional status (rehabilitation responder) was independently associated with weaning success or survival at hospital discharge or 3 months after RCC discharge. In patients requiring $\mathrm{PMV}$, the rehabilitation responders (pre-rehabilitation DEMMI $<20$ and post-rehabilitation DEMMI $\geq 20$ ) had similar weaning and survival rates as those with good functional status at RCC transfer (both pre- and post-rehabilitation DEMMI $\geq$ 20). This finding re-emphasized the importance of pulmonary rehabilitation and the response to this treatment in patients requiring PMV. The functional status after rehabilitation could be used as a surrogate marker for weaning and subsequent survival and it could assist both clinicians and families in understanding the prognosis during RCC stay, therefore avoiding futile aggressive treatment or introducing early palliative care. The benefit of rehabilitation may be related to treatment of muscle weakness and management of airway secretions, and therefore, improving respiratory function and decreasing bed rest complication (9). In patients with pre-rehabilitation DEMMI $<20$, the rehabilitation responders (post-rehabilitation DEMMI $\geq 20$ ) were more likely to have higher body mass index (OR, 1.108; 95\% CI, 1.029-1.193; $P=0.006)$ and higher Glasgow Coma Scale (OR, 1.691; 95\% CI, 1.274-2.245; $P<0.001)$ than the rehabilitation non-responders (post-rehabilitation DEMMI < 20) (Supplementary Table 4). Therefore, poor response to rehabilitation may be related to impaired nutritional or neurologic status in patients required PMV. Further researches are needed to elucidate whether response to rehabilitation could be modified by interventions targeting nutritional and neurologic components such as aggressive nutritional support and sedation/delirium protocol.

Besides weaning success and higher post-rehabilitation functional status, our study showed a higher platelet count was independently associated with both survival at hospital discharge and 3 months after RCC discharge. This finding was generally consistent with previous observational studies and meta-analysis $(3,27,28)$. The underlying cause of thrombocytopenia can be multifactorial (29). The association between thrombocytopenia and mortality in patients requiring PMV may just reflect unresolved underlying disease or acute critical condition, and prophylactic platelet transfusion may increase the risk of nosocomial infection, thrombotic events, organ dysfunction, and even mortality (30).

The present study had some limitations. First, this is a single center study with retrospective nature. Second, survival status beyond 3 months after RCC discharge was not obtained. Third, the average age of the population was more than 70 years, which was higher than that in previous PMV studies (31). Our results may not be generalizable to other population.

\section{CONCLUSIONS}

Functional status after pulmonary rehabilitation was independently associated with weaning success, hospital survival, and 3-month overall survival after RCC discharge in patients requiring PMV support. Post-rehabilitation functional status, but not pre-rehabilitation functional status, was a significant prognostic factor associated with weaning success and survival in patients requiring PMV. Physicians, therefore, should not decline or withhold rehabilitation for PMV patients who had poor performance status at initial post-ICU stage. Further studies were warranted to validate our findings.

\section{DATA AVAILABILITY STATEMENT}

The raw data supporting the conclusions of this article will be made available by the authors, without undue reservation. 


\section{ETHICS STATEMENT}

The studies involving human participants were reviewed and approved by The Institutional Review Board authority of National Taiwan University Hospital Hsin-Chu Branch (REC: 107-043-E). Written informed consent for participation was not required for this study in accordance with the national legislation and the institutional requirements.

\section{AUTHOR CONTRIBUTIONS}

L-TK, S-KL, C-PT, and M-RL participated in the study concept and design. C-PT, Y-FW, P-HT, C-HC, L-YC, and $\mathrm{K}-\mathrm{LY}$ participated in the collection of data, statistical analysis, interpretation of data, and manuscript draft. S-KL, M-RL, and

\section{REFERENCES}

1. Bellani G, Laffey JG, Pham T, Fan E, Brochard L, Esteban A, et al. Epidemiology, patterns of care, and mortality for patients with acute respiratory distress syndrome in intensive care units in 50 countries. JAMA. (2016) 315:788-800. doi: 10.1001/jama.2016.0291

2. Boles JM, Bion J, Connors A, Herridge M, Marsh B, Melot C, et al. Weaning from mechanical ventilation. Eur Respir J. (2007) 29:103356. doi: 10.1183/09031936.00010206

3. Dettmer MR, Damuth E, Zarbiv S, Mitchell JA, Bartock JL, Trzeciak S. Prognostic factors for long-term mortality in critically ill patients treated with prolonged mechanical ventilation: a systematic review. Crit Care Med. (2017) 45:69-74. doi: 10.1097/CCM.0000000000002022

4. Bigatello LM, Stelfox HT, Berra L, Schmidt U, Gettings EM. Outcome of patients undergoing prolonged mechanical ventilation after critical illness. Crit Care Med. (2007) 35:2491-7. doi: 10.1097/01.CCM.0000287589.16724.B2

5. Ambrosino N, Vitacca M. The patient needing prolonged mechanical ventilation: a narrative review. Multidiscip Respir Med. (2018) 13:6. doi: 10.1186/s40248-018-0118-7

6. Chiang LL, Wang LY, Wu CP, Wu HD, Wu YT. Effects of physical training on functional status in patients with prolonged mechanical ventilation. Phys Ther. (2006) 86:1271-81. doi: 10.2522/ptj.20050036

7. Chen YH, Lin HL, Hsiao HF, Chou LT, Kao KC, Huang CC, et al. Effects of exercise training on pulmonary mechanics and functional status in patients with prolonged mechanical ventilation. Respir Care. (2012) 57:72734. doi: 10.4187/respcare.01341

8. Chen S, Su CL, Wu YT, Wang LY, Wu CP, Wu HD, et al. Physical training is beneficial to functional status and survival in patients with prolonged mechanical ventilation. J Formos Med Assoc. (2011) 110:5729. doi: 10.1016/j.jfma.2011.07.008

9. Ambrosino N, Venturelli E, Vagheggini G, Clini E. Rehabilitation, weaning and physical therapy strategies in chronic critically ill patients. Eur Respir J. (2012) 39:487-92. doi: 10.1183/09031936.00094411

10. Clini EM, Crisafulli E, Antoni FD, Beneventi C, Trianni L, Costi S, et al. Functional recovery following physical training in tracheotomized and chronically ventilated patients. Respir Care. (2011) 56:306-13. doi: 10.4187/respcare.00956

11. MacIntyre NR, Epstein SK, Carson S, Scheinhorn D, Christopher K, Muldoon S. Management of patients requiring prolonged mechanical ventilation: report of a NAMDRC consensus conference. Chest. (2005) 128:393754. doi: 10.1378/chest.128.6.3937

12. de Morton NA, Davidson M, Keating JL. The de Morton Mobility Index (DEMMI): an essential health index for an ageing world. Health Qual Life Outcomes. (2008) 6:63. doi: 10.1186/1477-7525-6-63

13. Sommers J, Vredeveld T, Lindeboom R, Nollet F, Engelbert RH, van der Schaaf M. de Morton mobility index is feasible, reliable, and valid in patients with critical illness. Phys Ther. (2016) 96:1658-66. doi: 10.2522/ptj.20150339
J-CK participated in manuscript revision. All authors read and approved the final manuscript.

\section{ACKNOWLEDGMENTS}

The authors thank all the nursing staffs, respiratory therapists, and physical therapists at the RCC in National Taiwan University Hospital Hsin-Chu Branch for excellent patient care during the study period.

\section{SUPPLEMENTARY MATERIAL}

The Supplementary Material for this article can be found online at: https://www.frontiersin.org/articles/10.3389/fmed. 2021.675103/full\#supplementary-material

14. de Morton NA, Davidson M, Keating JL. Validity, Responsiveness and the minimal clinically important difference for the De Morton Mobility Index (DEMMI) in an older acute medical population. BMC Geriatr. (2010) 10:72. doi: 10.1186/1471-2318-10-72

15. New PW, Scroggie GD, Williams CM. The validity, reliability, responsiveness and minimal clinically important difference of the de Morton mobility index in rehabilitation. Disabil Rehabil. (2017) 39:1039-43. doi: 10.1080/09638288.2016.1179800

16. Trøstrup J, Andersen H, Kam CAM, Magnusson SP, Beyer N. Assessment of mobility in older people hospitalized for medical illness using the de Morton Mobility index and cumulated ambulation score-validity and minimal clinical important difference. J Geriatr Phys Ther. (2019) 42:15360. doi: 10.1519/JPT.0000000000000170

17. Rhodes A, Evans LE, Alhazzani W, Levy MM, Antonelli M, Ferrer R, et al. Surviving sepsis campaign: international guidelines for management of sepsis and septic shock: 2016. Crit Care Med. (2017) 45:486552. doi: 10.1097/CCM.0000000000002255

18. ARDS Definition Task Force, Ranieri VM, Rubenfeld GD, Thompson BT, Ferguson ND, Caldwell E, et al. Acute respiratory distress syndrome: the Berlin Definition. JAMA. (2012) 307:2526-33. doi: 10.1001/jama.2012.5669

19. Knaus WA, Draper EA, Wagner DP, Zimmerman JE. APACHE II: a severity of disease classification system. Crit Care Med. (1985) 13:818-29.

20. MacIntyre N. Discontinuing mechanical ventilatory support. Chest. (2007) 132:1049-56. doi: 10.1378/chest.06-2862

21. Vitacca M, Paneroni M, Bianchi L, Clini E, Vianello A, Ceriana P, et al. Maximal inspiratory and expiratory pressure measurement in tracheotomised patients. Eur Respir J. (2006) 27:343-9. doi: 10.1183/09031936.06.00002705

22. Shih CY, Hung MC, Lu HM, Chen L, Huang SJ, Wang JD. Incidence, life expectancy and prognostic factors in cancer patients under prolonged mechanical ventilation: a nationwide analysis of 5,138 cases during 1998-2007. Crit Care. (2013) 17:R144. doi: 10.1186/cc12823

23. Yang PH, Wang CS, Wang YC, Yang CJ, Hung JY, Hwang JJ, et al. Outcome of physical therapy intervention on ventilator weaning and functional status. Kaohsiung J Med Sci. (2010) 26:366-72. doi: 10.1016/S1607-551X(10)7 0060-7

24. Keng LT, Chung KP, Lin SY, Liang SK, Cheng JC, Chen IC, et al. Significant clinical factors associated with long-term mortality in critical cancer patients requiring prolonged mechanical ventilation. Sci Rep. (2017) 7:2148. doi: 10.1038/s41598-017-02418-4

25. Hill K, Dennis DM, Patman SM. Relationships between mortality, morbidity, and physical function in adults who survived a period of prolonged mechanical ventilation. J Crit Care. (2013) 28:427-32. doi: 10.1016/j.jcrc.2013.02.012

26. Carson SS, Bach PB, Brzozowski L, Leff A. Outcomes after long-term acute care. An analysis of 133 mechanically ventilated patients. Am J Respir Crit Care Med. (1999) 159:1568-73. doi: 10.1164/ajrccm.159.5.98 09002 
27. Carson SS, Garrett J, Hanson LC, Lanier J, Govert J, Brake MC, et al. A prognostic model for one-year mortality in patients requiring prolonged mechanical ventilation. Crit Care Med. (2008) 36:2061-9. doi: 10.1097/CCM.0b013e31817b8925

28. Hough CL, Caldwell ES, Cox CE, Douglas IS, Kahn JM, White DB, et al. Development and validation of a mortality prediction model for patients receiving 14 days of mechanical ventilation. Crit Care Med. (2015) 43:233945. doi: 10.1097/CCM.0000000000001205

29. Greinacher A, Selleng S. How I evaluate and treat thrombocytopenia in the intensive care unit patient. Blood. (2016) 128:303242. doi: 10.1182/blood-2016-09-693655

30. Blumberg N, Cholette JM, Schmidt AE, Phipps RP, Spinelli SL, Heal JM, et al. Management of platelet disorders and platelet transfusions in ICU patients. Transfus Med Rev. (2017) 31:252-7. doi: 10.1016/j.tmrv.2017. 04.002

31. Damuth E, Mitchell JA, Bartock JL, Roberts BW, Trzeciak S. Long-term survival of critically ill patientstreated with prolonged mechanical ventilation: a systematic review and meta-analysis. Lancet Respir Med. (2015) 3:54453. doi: 10.1016/S2213-2600(15)00150-2

Conflict of Interest: S-KL received speaking honoraria from Norvatis, Pfizer, Roche, and Boehringer Ingelheim.

The remaining authors declare that the research was conducted in the absence of any commercial or financial relationships that could be construed as a potential conflict of interest.

Copyright (C) 2021 Keng, Liang, Tseng, Wen, Tsou, Chang, Chang, Yu, Lee and Ko. This is an open-access article distributed under the terms of the Creative Commons Attribution License (CC BY). The use, distribution or reproduction in other forums is permitted, provided the original author(s) and the copyright owner(s) are credited and that the original publication in this journal is cited, in accordance with accepted academic practice. No use, distribution or reproduction is permitted which does not comply with these terms. 